\title{
Analytical theory of the motion of Phobos: A comparison with numerical integration
}

\author{
P. Wa̧ż $\dot{Z}^{\star}$ \\ Torun Centre for Astronomy of the Nicolaus Copernicus University, Gagarina 11, 87-100 Torun, Poland
}

Received 30 August 2002 / Accepted 25 November 2003

\begin{abstract}
A new theory of the motion of Phobos - a moon of Mars - has been presented. The theory is based on the problem of two fixed gravitational centers. The analytical functions appearing in this model are expressed as a series of the third order with respect to the $J_{2}$ and $\frac{J_{3}}{J_{2}}$ zonal harmonics of Mars. The interaction between Phobos and Mars is described by a potential consisting of very many elements. The zonal harmonics of order $\leq 12$ and tesseral harmonics of order $\leq 6$ are taken into account. Interactions of Phobos with the Sun, Jupiter, Deimos - another moon of Mars, and the tidal potential of the Sun are also taken into account.
\end{abstract}

Key words. planets and satellites: individual: Phobos - methods: analytical

\section{Introduction}

In the next two decades one should expect several missions to the vicinity of Mars aimed mainly at a preparation for the landing of humans on this planet. Therefore a precise knowledge of the phenomena that may influence the motion of all objects in the proximity of Mars is of particular interest and importance. In particular, a good analytical model of the motion of the Martian satellites may be used as a starting point for a precise description of other objects, e.g. of the artificial satellites of Mars that will be launched in the future. Recently a new analytical model of the motion of Phobos has been developed by the present author (Wạż 1999a). The theory is based on the problem of two fixed gravitational centers. The Hamiltonian, apart from the integrable part describing the problem of two fixed centers, contains perturbative terms which include perturbations due to the Sun as well as perturbations connected with zonal harmonics $j_{4}-j_{12}$ and with sectorial-tesseral harmonics of order 6. Also the influences of the tidal interactions of the Sun and of Jupiter and Deimos on the motion of Phobos have been taken into account.

In the present paper the results obtained in the process of the analytical integration of the Hamilton equations of motion using the previously defined Hamiltonian (Wa̧ż 1999b) are described. The formulae describing the secular and periodic perturbations in Euler's orbit of Phobos are also presented. To estimate the precision of the analytical formulae, the final analytical solution has been compared with the results of numerical integration.

\footnotetext{
^ e-mail:piotr.waz@astri.uni.torun.pl
}

\section{Disturbing function}

As a consequence of an analysis of the values of the appropriate components of the gravitational potential of Phobos, the Hamilton function has been constructed in the following way:

$K=K_{0}+K_{\mathrm{p}}$,

where

$K_{\mathrm{p}}=K_{\mathrm{p}}^{\mathrm{zh}}+K_{\mathrm{p}}^{\mathrm{th}}+K_{\mathrm{p}}^{\mathrm{S}}+K_{\mathrm{p}}^{\mathrm{D}}+K_{\mathrm{p}}^{\mathrm{J}}+K_{\mathrm{p}}^{\mathrm{t}}+K_{\mathrm{p}}^{\mathrm{pr}}$.

$K_{0}$ describes the problem of two fixed gravitational centers. $K_{\mathrm{p}}$ is the perturbational function of the analytically solvable problem $K_{0}$. The function $K_{\mathrm{p}}$ consists of the components generated by the zonal harmonics from $J_{4}$ up to $J_{12}\left(K_{\mathrm{p}}^{\mathrm{zh}}\right)$, sectorial-tesseral harmonics of order $6\left(K_{\mathrm{p}}^{\mathrm{th}}\right)$. The influence of the Sun $\left(K_{\mathrm{p}}^{\mathrm{S}}\right)$, Deimos $\left(K_{\mathrm{p}}^{\mathrm{D}}\right)$ and Jupiter $\left(K_{\mathrm{p}}^{\mathrm{J}}\right)$ are also important factors for the motion of Phobos and have been taken into account in the theory. The tidal effects of the Sun $\left(K_{\mathrm{p}}^{\mathrm{t}}\right)$ and the fact that the chosen coordinate system is noninertial $\left(K_{\mathrm{p}}^{\mathrm{pr}}\right)$ also have been considered in the theory. Each component of the perturbational function has been presented using the osculating variables of the problem of two fixed gravitational centers $(a, e$, $s, l, g, h)$. The variables describe the semi-major axis, eccentricity, the sine of the inclination of the orbit to the coordinate system, the mean anomaly, the argument of perihelium and the longitude of the node for the Euler's orbit (the orbit defined for the general problem of the two fixed gravitational centers), respectively. This orbit has been defined in the coordinate system in which the main $0 x y$ plane has been related to the mean equator of date of Mars $\left(R_{\mathrm{M}}\right)$. The $0 x$ axis has been directed to 
the breaking points of $R_{\mathrm{M}}$ with the mean orbit of date of Mars. The $0 z$ axis has been chosen in such a way that the coordinate system is righthanded (Wạż 1999a). Under the conditions described above, the Hamilton function consists of 22000 terms:

$$
\begin{aligned}
& \sum_{n} C \varepsilon^{2 k_{1}} \varepsilon \sigma^{k_{2}} v^{k_{3}} \mu^{k_{4}} e^{k_{e}} s^{k_{s}} a^{k_{a}} \sin _{\cos }\left(k_{l} l+k_{g} g+k_{h} h+k_{l \mathrm{~J}} l_{\mathrm{J}}\right. \\
& \left.\quad+k_{l \mathrm{~S}} l_{\mathrm{S}}+k_{\omega \mathrm{S}} \omega_{\mathrm{S}}+k_{l \mathrm{D}} l_{\mathrm{D}}+k_{\Omega \mathrm{D}} \Omega_{\mathrm{D}}+k_{\omega \mathrm{D}} \omega_{\mathrm{D}}+k_{G t} G_{t}\right),
\end{aligned}
$$

where

$$
\begin{aligned}
n \equiv & k_{e}, k_{s}, k_{a}, k_{l}, k_{g}, k_{h}, k_{l \mathrm{~J}}, k_{l \mathrm{~S}}, k_{\omega \mathrm{S}}, \\
& k_{l \mathrm{D}}, k_{\omega \mathrm{D}}, k_{\Omega \mathrm{D}}, k_{G t}, k_{1}, k_{2}, k_{3}, k_{4}, \\
& k_{1}+k_{2}+k_{3}+k_{4}=1, \\
v= & \frac{3}{4} \varepsilon^{2}\left(4-5 s^{2}\right), \quad \mu=\varepsilon^{2}\left(1+\frac{1}{2} e^{2}\right) \sqrt{1-s^{2}}, \\
\varepsilon^{2}= & \frac{c^{2}}{a^{2}\left(1-e^{2}\right)^{2}}, \quad \varepsilon \sigma=\frac{c \sigma}{a\left(1-e^{2}\right)}, \\
c= & r_{0} \sqrt{J_{2}-\left(\frac{J_{3}}{2 J_{2}}\right)^{2}}, \\
\sigma= & \frac{J_{3}}{2 J_{2} \sqrt{J_{2}-\left(\frac{J_{3}}{2 J_{2}}\right)^{2}}} .
\end{aligned}
$$

$r_{0}$ is the equatorial radius of the central mass, $l_{\mathrm{J}}$ is the mean anomaly of Jupiter, $l_{\mathrm{S}}$ is the mean anomaly of Mars, $\omega_{\mathrm{S}}=\omega_{\mathrm{M}}-$ $\Omega_{\mathrm{M}}-Q+180^{\circ}\left(\omega_{\mathrm{M}}-\right.$ the longitude of the perihelion of Mars, $\Omega_{\mathrm{M}}$ - the longitude of the ascending node of the Mars orbit, $Q$ - the angle between $O x$ axis and the line connecting the center of the reference frame with the point where the ecliptic crosses with the Mars orbit), $l_{\mathrm{D}}, \omega_{\mathrm{D}}$ and $\Omega_{\mathrm{D}}$ are the mean anomaly, the longitude of the node and the argument of the pericentre of the Deimos orbit. $G_{t}$ is the sidereal time defined for Mars. The coefficients $k_{e}, k_{s}, k_{a}, k_{l}, k_{g}, k_{h}, k_{l \mathrm{~J}}, k_{l \mathrm{~S}}, k_{\omega \mathrm{S}}, k_{l \mathrm{D}}, k_{\omega \mathrm{D}}, k_{\Omega \mathrm{D}}$, $k_{G t}$, are the numerical values depending on the accuracy of the expanding perturbational function (see Wa̧ż 1999a). The values describing the orbits of Phobos and Deimos have been taken from Emelianov et al. (1993) and the parameters related to the orbit of Mars and Jupiter from Bretagnon (1982). The values of the particular components of the gravitational field of Mars have been taken from Konopliv \& Sjogren (1995).

\section{Averaging of the equations of motion}

After the construction of the Hamilton function, the next step is solving the system of equations describing the motion of Phobos:

$$
\begin{array}{lll}
\frac{\mathrm{d} l}{\mathrm{~d} t}=-\frac{\partial K}{\partial L}, & \frac{\mathrm{d} g}{\mathrm{~d} t}=-\frac{\partial K}{\partial G}, & \frac{\mathrm{d} h}{\mathrm{~d} t}=-\frac{\partial K}{\partial H}, \\
\frac{\mathrm{d} L}{\mathrm{~d} t}=\frac{\partial K}{\partial l}, & \frac{\mathrm{d} G}{\mathrm{~d} t}=\frac{\partial K}{\partial g}, & \frac{\mathrm{d} H}{\mathrm{~d} t}=\frac{\partial K}{\partial h} .
\end{array}
$$

The system of these differential equations is nonintegrable. To solve the analytical problem the method of Lie-Hori, algorithmized by Mersman (1970), has been used. In this approach a new set of variables is defined. The relations between the new variables $\boldsymbol{q}, \boldsymbol{p}\left(\boldsymbol{q}=\left(q_{1}, q_{2}, \ldots, q_{n}\right)\right.$, $\left.\boldsymbol{p}=\left(p_{1}, p_{2}, \ldots, p_{n}\right)\right)$ and the old ones $\boldsymbol{x}, \boldsymbol{y}(\boldsymbol{x}=$ $\left.\left(x_{1}, x_{2}, \ldots, x_{n}\right), \boldsymbol{y}=\left(y_{1}, y_{2}, \ldots, y_{n}\right)\right)$ are described by a system of differential equations

$\frac{\mathrm{d} x_{i}}{\mathrm{~d} \tau}=\frac{\partial W(\boldsymbol{x}, \boldsymbol{y})}{\partial y_{i}}, \quad \frac{\mathrm{d} y_{i}}{\mathrm{~d} \tau}=-\frac{\partial W(\boldsymbol{x}, \boldsymbol{y})}{\partial x_{i}}, \quad i=1,2, \ldots, n$

with the initial conditions

$x_{i}=q_{i}, \quad y_{i}=p_{i}$, for $\tau=0$,

where $W$ is the generating function of the transformation and the independent variable $\tau$ takes values in the interval $\langle 0, \epsilon>$.

An arbitrary function $F(\boldsymbol{x}, \boldsymbol{y})$ dependent on the old variables, after the transformation to the new ones, may be evaluated using the relation:

$$
F(\boldsymbol{x}, \boldsymbol{y})=\left.\sum_{n=0}^{\infty} \frac{\epsilon^{n}}{n !} \frac{\mathrm{d}^{n} F(\boldsymbol{x}, \boldsymbol{y})}{\mathrm{d} \tau^{n}}\right|_{\tau=0 ;(\boldsymbol{x}, \boldsymbol{y})=(\boldsymbol{q}, \boldsymbol{p})}=\sum_{n=0}^{\infty} \frac{\epsilon^{n}}{n !} D^{n} F .
$$

$\epsilon$ is an auxiliary parameter to determine which of the components has to be taken into account when a specific order of the Poisson bracket is evaluated.

The Lie operator $D$ is defined as

$D^{0} F \equiv F$,

$D^{n+1} F=D D^{n} F, \quad n=1,2,3 \ldots$,

where

$D F \equiv\{F, W\}=\sum_{i=0}^{n}\left(\frac{\partial F}{\partial y_{i}} \frac{\partial W}{\partial x_{i}}-\frac{\partial F}{\partial x_{i}} \frac{\partial W}{\partial y_{i}}\right)$.

In the new variables the Hamilton function $E$ depends on neither the angular variables nor on the time. Using the averaged equations one can obtain the secular perturbations using the elements $l^{\prime}, g^{\prime}, h^{\prime}$ :

$\frac{\mathrm{d} l^{\prime}}{\mathrm{d} t}=-\frac{\partial E}{\partial L^{\prime}}$

$\frac{\mathrm{d} g^{\prime}}{\mathrm{d} t}=-\frac{\partial E}{\partial G^{\prime}}$

$\frac{\mathrm{d} h^{\prime}}{\mathrm{d} t}=-\frac{\partial E}{\partial H^{\prime}}$.

Taking the values used to cut the series of the perturbational functions, one can express the secular changes in degrees per year. The results are collected in Tables 1 and 2 .

$j_{4}-j_{12}$ are the differences between the true zonal harmonics of Mars $\left(J_{4}-J_{12}\right)$ and values of zonal harmonics created by the potential of two fixed gravitational centers.

The values of the secular perturbations which result from $j_{7}$ and from $j_{5}$ harmonics ${ }^{1}$ are particularly interesting. These values are of a similar order of magnitude as the perturbations caused by $j_{12}$, by the influence of Deimos and by the tidal effects of the Sun. The first-order perturbational function vanishes. However, this does not imply that there are no first-order terms in the secular perturbations. Simply the first-order terms

\footnotetext{
${ }^{1}$ It is the combination of $j_{5}$ and of a term proportional to $J_{3} / J_{2}$.
} 
Table 1. Secular perturbations connected to appropriate parts of the perturbational function (all values are in degrees per year).

\begin{tabular}{lrrr}
\hline \hline part of $K_{\mathrm{p}}$ & \multicolumn{1}{c}{$\mathrm{d} l / \mathrm{d} t$} & \multicolumn{1}{c}{$\mathrm{d} g / \mathrm{d} t$} & \multicolumn{1}{c}{$\mathrm{d} h / \mathrm{d} t$} \\
\hline Sun & $-1.1463006017044 \times 10^{-1}$ & $9.816816540467 \times 10^{-2}$ & $-4.911475140914 \times 10^{-2}$ \\
$j_{4}$ & $-5.79638508 \times 10^{-6}$ & $6.4502793982957 \times 10^{-1}$ & $-3.2261644032716 \times 10^{-1}$ \\
$j_{5}$ & $3.4581041880 \times 10^{-4}$ & $-6.9298586158 \times 10^{-4}$ & $3.4756297072 \times 10^{-4}$ \\
$j_{6}$ & $1.066785850750 \times 10^{-2}$ & $-6.414029270089 \times 10^{-2}$ & $3.210311858491 \times 10^{-2}$ \\
$j_{7}$ & $4.176573126 \times 10^{-5}$ & $-8.385050563 \times 10^{-5}$ & $4.214447247 \times 10^{-5}$ \\
$j_{8}$ & $-1.22792672086 \times 10^{-3}$ & $4.92764469606 \times 10^{-3}$ & $-2.46884614696 \times 10^{-3}$ \\
$j_{9}$ & $8.09601805 \times 10^{-6}$ & $-1.629375365 \times 10^{-5}$ & $8.21187001 \times 10^{-6}$ \\
$j_{10}$ & $8.1450458110 \times 10^{-4}$ & $-2.72788657345 \times 10^{-3}$ & $1.36851428880 \times 10^{-3}$ \\
$j_{11}$ & $-1.94681243 \times 10^{-6}$ & $3.93012459 \times 10^{-6}$ & $-1.98734613 \times 10^{-6}$ \\
$j_{12}$ & $-1.1762943455 \times 10^{-4}$ & $3.5520395270 \times 10^{-4}$ & $-1.7848397333 \times 10^{-4}$ \\
$K_{\mathrm{p}}^{\mathrm{pr}}$ & 0 & 0 & $1.76674629326 \times 10^{-3}$ \\
$K_{\mathrm{p}}^{\mathrm{t}}$ & $1.530976715 \times 10^{-5}$ & $3.059290224 \times 10^{-5}$ & $-1.530931493 \times 10^{-5}$ \\
$K_{\mathrm{p}}^{\mathrm{D}}$ & $-1.0117760802 \times 10^{-4}$ & $2.0242008603 \times 10^{-4}$ & $-1.0125542534 \times 10^{-4}$ \\
$K_{\mathrm{p}}^{\mathrm{J}}$ & $-3.29522891 \times 10^{-6}$ & $2.82173118 \times 10^{-6}$ & $-1.41004052 \times 10^{-6}$ \\
\hline
\end{tabular}

Table 2. Secular perturbations connected to appropriate orders of perturbations (all values are in degrees per year).

\begin{tabular}{lrrr}
\hline \hline $\begin{array}{l}\text { order of } \\
\text { the perturbation }\end{array}$ & $\mathrm{d} l / \mathrm{d} t$ & $\mathrm{~d} g / \mathrm{d} t$ & \multicolumn{1}{c}{$\mathrm{d} h / \mathrm{d} t$} \\
\hline zero & 412310.3265038 & 317.9067883 & -158.9673155 \\
first & 0 & 0 & 0 \\
second & $-1.03967998048 \times 10^{-1}$ & $6.79055812533 \times 10^{-1}$ & $-3.39628073151 \times 10^{-1}$ \\
third & $-2.26489288 \times 10^{-4}$ & $2.001596798 \times 10^{-3}$ & $7.65887648 \times 10^{-4}$ \\
total & 412310.2223093 & 318.5878458 & -159.3061777 \\
\hline
\end{tabular}

related to the mean orbit are included in the zero-order part of the Hamiltonian.

For an estimation of the periodic perturbations, the transformation function $W$ has been used. Using this function the relations between the variables describing the real motion $(l, g$, $h, L, G, H)$ and the averaged variables $\left(l^{\prime}, g^{\prime}, h^{\prime}, L^{\prime}, G^{\prime}, H^{\prime}\right)$ have been found. The Poincaré coordinates have been introduced into the procedure for the estimation of the dependence on the average variables:

$$
\begin{array}{ll}
D_{1}=L, & d_{1}=l+g+h, \\
D_{2}=\sqrt{2(L-G)} \cos (g+h), & d_{2}=-\sqrt{2(L-G)} \sin (g+h), \\
D_{3}=\sqrt{2(G-H)} \cos (h), & d_{3}=-\sqrt{2(G-H)} \sin (h) .
\end{array}
$$

These coordinates have been chosen because they are nonsingular if the eccentricities and inclinations are small. The canonical transformation is described by the relations:

$p=x+x_{1}(x, y)+x_{2}(x, y)+x_{3}(x, y)+\cdots$

$$
q=y+y_{1}(x, y)+y_{2}(x, y)+x_{3}(x, y)+\cdots,
$$

where

$$
\begin{aligned}
& x_{1}=\left\{\boldsymbol{x}, W_{1}\right\}, \\
& y_{1}=\left\{\boldsymbol{y}, W_{1}\right\}, \\
& \boldsymbol{x}_{2}=\frac{1}{2}\left\{\left\{\boldsymbol{x}, W_{1}\right\}, W_{1}\right\}+\left\{\boldsymbol{x}, W_{2}\right\}, \\
& \boldsymbol{y}_{2}=\frac{1}{2}\left\{\left\{\boldsymbol{y}, W_{1}\right\}, W_{1}\right\}+\left\{\boldsymbol{y}, W_{2}\right\},
\end{aligned}
$$

$$
\begin{aligned}
\boldsymbol{x}_{3}= & \frac{1}{6}\left\{\left\{\left\{\boldsymbol{x}, W_{1}\right\}, W_{1}\right\}, W_{1}\right\}+\frac{1}{2}\left\{\left\{\boldsymbol{x}, W_{2}\right\}, W_{1}\right\} \\
& +\frac{1}{2}\left\{\left\{\boldsymbol{x}, W_{1}\right\}, W_{2}\right\}+\left\{\boldsymbol{x}, W_{3}\right\}, \\
\boldsymbol{y}_{3}= & \frac{1}{6}\left\{\left\{\left\{\boldsymbol{y}, W_{1}\right\}, W_{1}\right\}, W_{1}\right\}+\frac{1}{2}\left\{\left\{\boldsymbol{y}, W_{2}\right\}, W_{1}\right\} \\
& +\frac{1}{2}\left\{\left\{\boldsymbol{y}, W_{1}\right\}, W_{2}\right\}+\left\{\boldsymbol{y}, W_{3}\right\},
\end{aligned}
$$

$\boldsymbol{y}=\left(D_{1}, D_{2}, D_{3}\right)$ and $\boldsymbol{x}=\left(d_{1}, d_{2}, d_{3}\right)$ are the series describing the periodic changes in the elements $d_{1}, d_{2}, d_{3}, D_{1}, D_{2}, D_{3}$.

$W_{1}, W_{2}, W_{3}$ are the generating functions of the transformations of order one, two and three respectively. The spectra of the periodic changes of the Poincaré coordinates are shown in Fig. 1. The abscissae are equal to the derivatives of the argument of the appropriate trigonometric function $T$ (expressed in the figures in radians per days) and the coordinates are equal to the logarithm of the corresponding amplitude $A$ expressed in the units of the theory ( $r_{0}$ denotes the radius of Mars).

As one can see, the major part of the spectrum of $D_{1}$ is located in the area of short periods. The changes of this variable correspond to the changes of the semi-major axis. The largest amplitudes are multiplied by the trigonometrical functions with arguments $l+g+h-g_{t}, 2 l+2 h+2 g-2 g_{t}, 3 l+2 h+2 g-2 g_{t}$, $3 l+3 g+3 h-3 g_{t}$ and $4 l+4 g+4 h-4 g_{t}$. The elements responsible for the perturbations due to Deimos are located in the left-most part of the spectrum.

The changes of $d_{1}$ are the ones in the mean longitude. Particularly large amplitudes are connected to the arguments of 

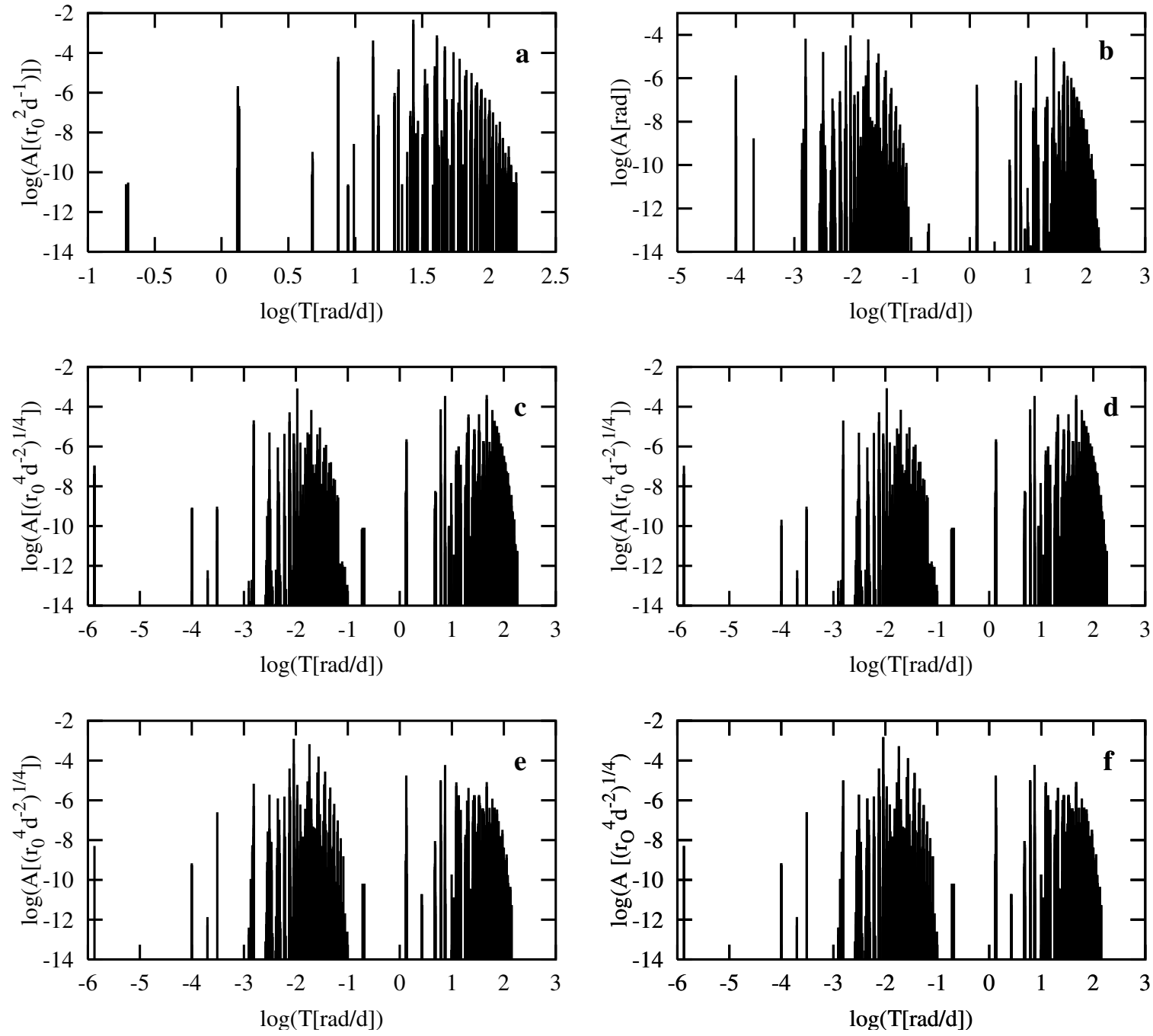

Fig. 1. Spectra of the periodic perturbations of the variables: a) $D_{1}$, b) $d_{1}$, c) $D_{2}$, d) $d_{2}$, e) $D_{3}$,f) $d_{3}$.

the function equal to $2 l+2 g+2 h-2 g_{t}, l_{\mathrm{M}}, 2 l_{\mathrm{M}}+2 \tilde{\omega}_{\mathrm{S}}, 3 l_{\mathrm{M}}+2 \tilde{\omega}_{\mathrm{S}}$, $2 g+2 h-2 l_{\mathrm{M}}-2 \tilde{\omega}_{\mathrm{S}}, l+g+h$ and $h+l_{\mathrm{M}}$.

The spectra of $D_{2}$ and $d_{2}$ together present the periodic changes of the eccentricity and of the longitude of the pericentrum (defined for the problem of two fixed gravitational centers). In these variables the dominant amplitudes are connected to the sine and cosine function arguments equal to: $l+g+h-2 g_{t}$, $3 l+3 g+3 h-2 g_{t}, g+h-2 l_{\mathrm{M}}-2 \tilde{\omega}_{\mathrm{S}}$.

The changes of the inclination and of the longitude of the node are connected to $D_{3}$ and $d_{3}$. The elements connected to the influence of the Sun generate in these coordinates the highest amplitudes. The arguments of these terms are equal to $l_{\mathrm{M}}, 2 l_{\mathrm{M}}+$ $2 \tilde{\omega}_{\mathrm{S}}$ and $3 l_{\mathrm{M}}+2 \tilde{\omega}_{\mathrm{S}}$.

As can be seen from the figures, the same terms that are the most important in the perturbational function are also dominant in the spectra. The most important are the terms connected to $J_{22}$ and the perturbations due to the Sun. In all the spectra, except the coordinates $D_{1}$ and $d_{1}$, one can find a term with a long period $\left(1.33 \times 10^{-6} \mathrm{rad} \mathrm{d}^{-1}\right)$. This period corresponds to the time derivative for the argument $g+2 h$.

\section{Test of accuracy of the theory}

A comparison of the solutions derived in the analytical way with the ones obtained by a numerical integration of the equations of the motion is a good test of accuracy of the analytical theory. The main part of the numerical integration code constitutes the procedure RA15 (Everhart 1974). The code performs the integration of six differential equations for the rectangular components of the positions and velocities of Phobos. The coordinate system of the analytical theory has been used. All forces that have been taken into account in the analytical theory are also included in the numerical calculations. The interval of time of the integration is 2000 days. This time interval corresponds to two periods of the smallest angular variable describing the motion of Phobos.

The initial conditions have been derived from the expansions obtained using the analytical theory. 500 osculating elements, uniformly described along the Phobos orbit have been determined at the considered integral interval (using the numerical method). These values have been compared with the 

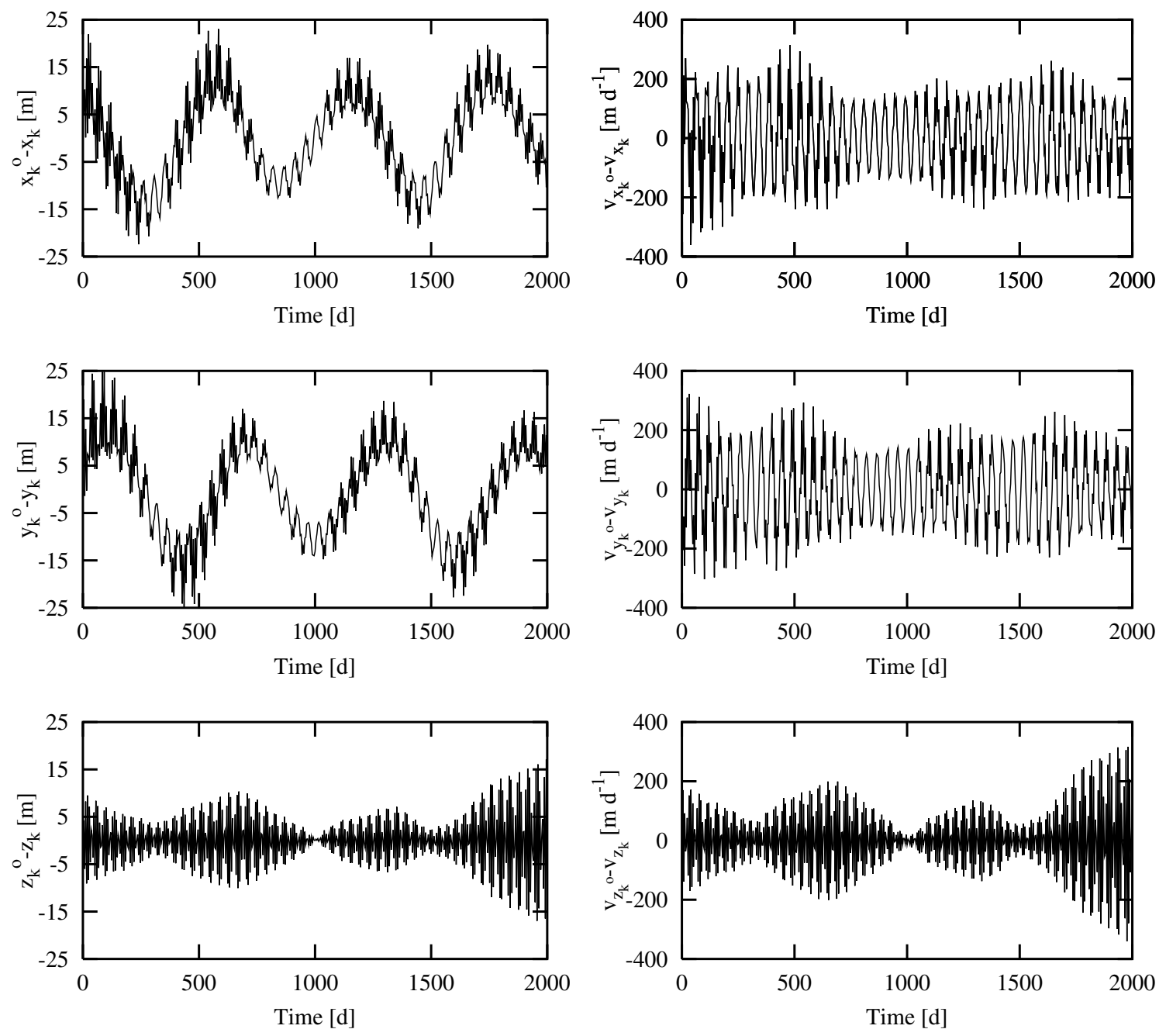

Fig. 2. Differences between the analytical theory and numerical integration for components of the position vectors $\left(x_{k}, y_{k}, z_{k}\right)$ and for velocities $\left(V_{x_{k}}, V_{y_{k}}, V_{z_{k}}\right)$.

osculating elements predicted by the analytical theory. The analytical osculating elements $\left(a, e, i, l_{0}, g_{0}, h_{0}\right)$ have been calculated as sums of the mean values for a given time $\left(a^{(0)}, e^{(0)}, i^{(0)}\right.$, $\left.l_{0}^{(0)}, g_{0}^{(0)}, h_{0}^{(0)}\right)$ and of the periodic terms $(\delta a, \delta e, \delta i, \delta l, \delta g, \delta h)$ which depend on these mean values.

$a=a^{(0)}+\delta a, \quad l_{0}=l_{0}^{(0)}+\delta l$,

$e=e^{(0)}+\delta e, \quad g_{0}=g_{0}^{(0)}+\delta g$,

$i=i^{(0)}+\delta i, \quad h_{0}=h_{0}^{(0)}+\delta h$.

For a given moment of time $\delta a, \delta e, \delta i, \delta l, \delta g, \delta h$ are evaluated and, as a consequence, the perturbed elements of the intermediate orbit can be determined.

Finally, using the relations between the elements of the Euler orbit and the Cartesian coordinates (Aksenov et al. 1988) the Cartesian coordinates and the respective components of the velocities for a given moment of time are determined.

A direct comparison of the positions and velocities obtained by numerical integration and derived from the analytical expressions does not give any real information about the accuracy of the theory. This is because the analytical formulae always introduce a small error in the coordinates and in the velocities. This error influences the values of the mean motion. The difference between the mean motions derived from the analytical and from the numerical formulae introduces a secular deviation in the longitude which increases with increasing difference $t-t_{0}$. Therefore, another comparison of the two kinds of data must be used. Namely, the positions and the velocities derived from the numerical integration are treated as observational data. The coordinates and the velocities are (known) functions of the elements of the mean orbit (Aksenov 1977). The elements of the mean orbit derived in the analytical way are treated as a set of approximate elements. Using the data obtained from the numerical integration, the elements of the mean orbit are corrected. Consequently, the new starting values $a$ are found. In the next step, the positions and the velocities are found analytically, for each time point, using the new initial values. The Levenberg-Marquardt procedure (Press et al. 1992) has been used to correct the elements of the mean orbit.

The accuracy of the solutions has been tested for the period of 2000 days. This interval of time has been divided to 500 equally-spaced time points which cover the orbit of Phobos in a uniform way. The results of this procedure are displayed in Figs. 2 and 3. 


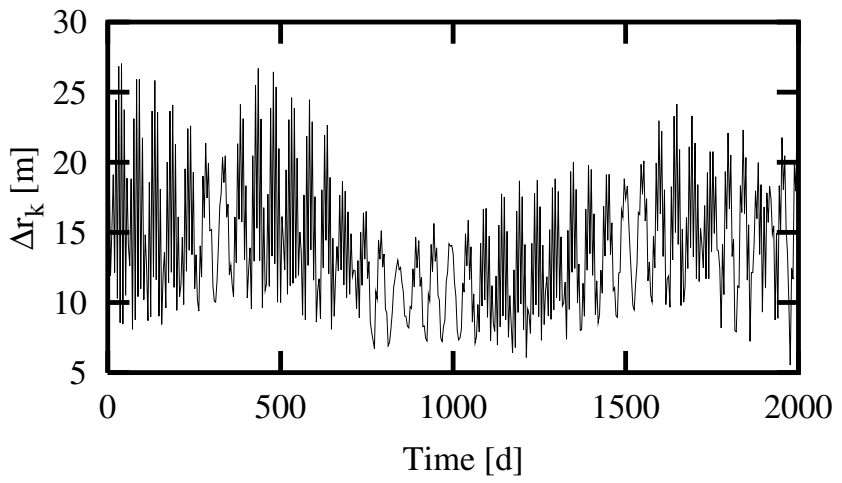

Fig. 3. Differences between the analytical theory and numerical integration for the vectors of the positions.

The differences between the position vectors derived from the numerical integration and the ones obtained from the analytical formulae are not larger than $30 \mathrm{~m}$. The error defined by the equation

$\varsigma=\sqrt{\frac{\sum_{k=0}^{m} \Delta r_{k}^{2}}{m+1},}$

where

$\Delta r_{k}=\sqrt{\left(x_{k}^{o}-x_{k}\right)^{2}+\left(y_{k}^{o}-y_{k}\right)^{2}+\left(z_{k}^{o}-z_{k}\right)^{2}}, \quad k=0, \ldots, m$

is not larger than $15 \mathrm{~m}$.
One should remember that these values do not describe the absolute accuracy of the analytical formulae. They have been obtained for given moments of time and a given time interval.

Acknowledgements. This work has been supported by the Polish KBN, project No. 5 PO3D 01221.

\section{References}

Aksenov, E. P. 1977, Theory of the Motion of Artificial Earth Satellite (Moskwa: Nauka)

Aksenov, E. P., Emeliannov, N. V., \& Tamarov, V. A. 1988, TGISh, 59,3

Bretagnon, P. 1982, A\&A, 114, 278

Emelianov, N. V., Vashkovyak, S. N., \& Nasonova, L. P. 1993, A\&A, 267,634

Everhart, E. 1974, Celest. Mech., 10, 35

Konopliv, A. S., \& Sjogren, W. L. 1995, JPL Publ., 95-5

Mersman, W. A. 1970, Celest. Mech., 3, 81

Wąż, P. 1999a, A\&A, 348, 300

Wạż, P. 1999b, Rep. Math. Phys., 44, 283

Press, W. H., Teukolsky, S. A., Vetterling, W. T., \& Flannery, B. P. 1992, Numerical Recipes in C. The Art of Scientific Computing (Cambridge: Univ. Press) 